\title{
LINKEDIN TALENT INSIGHTS FOR SOCIAL HIRING IN HR RECRUITMENT USING DATA MINING TECHNIQUES USING SOCIAL, MOBILITY, ANALYTICS, CLOUD (SMAC) ARCHITECTURE
}

\author{
Gayathri Denis \\ Research Scholar, Bharath Institute of Higher Education and Research (BIHER), \\ Chief Executive Officer, Ashish Infominds, Chennai, India

\section{Dr. T. Krishna Kumar} \\ Assistant Professor, Department of Computer Science and Engineering, \\ Bharath Institute of Higher Education and Research (BIHER), Chennai, India

\section{Dr. S. Sasipriya} \\ Professor, Department of Computer Science and Engineering, \\ Sri Krishna College Of Engineering and Technology, Coimbatore, India

\section{Dr. Karthikeyan} \\ Professor and Principal, Department of Computer Science and Engineering, \\ Tamilnadu College of Engineering, Coimbatore, India
}

\begin{abstract}
LinkedIn Talent Insights will enable enrollment specialists to look by aptitudes, area, and even school, social enlisting (otherwise called social procuring and web based life enlistment) is utilizing electronic life stages, for instance, Twitter and LinkedIn to expose occupations, find capacity, and talk with potential chooses about association culture. It is compelling for discovering latent applicants. This paper presents the Future trends in recruitment with SMAC - social, versatile, examination and cloud technology on one incorporated stack, where each capacity empowers another to augment their impact. This is the new endeavor IT model conveying an association that is increasingly connective, shared, continuous and profitable. The model is anticipated to improve business aggressiveness. The cooperative energy of all these four advancements develops advancement with low overheads and high reach. SMAC, is making another period where the conventional strategies are being supplanted by the cutting edge ones. So as to remain refreshed with current innovation and drive income through the computerized eco-space, SMAC is picking up stimulus making new position openings, reforming how trade happens and changing how rivalry influences the market.
\end{abstract}


Linkedin Talent Insights for Social Hiring in HR Recruitment using Data Mining Techniques using Social, Mobility, Analytics, Cloud (SMAC) Architecture

Keywords: Linkedin, Social Hiring, HR Recruitment, Data Mining, Analytics, SMAC

Cite this Article: Gayathri Denis, Dr. T. Krishna Kumar, Dr. Karthikeyan and Dr. S. Sasipriya, Linkedin Talent Insights for Social Hiring in HR Recruitment using Data Mining Techniques using Social, Mobility, Analytics, Cloud (SMAC) Architecture, International Journal of Advanced Research in Engineering and Technology (IJARET), 11 (1), 2020, pp 80-89.

http://iaeme.com/Home/issue/IJARET?Volume=11\&Issue $=1$

\section{INTRODUCTION}

Information mining may be a reasonably arrangement strategy that is admittedly accustomed concentrate hid examples from vast databases. data processing ideas and techniques will be applied in several fields like promoting, medication, land, consumer relationship the board, designing, web mining, so forth. unit of time enlisting exploitation data processing techniques combined with AI, quality \& Social Media may be a new rising technique of knowledge mining that may be applied on the info associated with job seekers. It utilizes varied procedures, as an example, selection trees, neural systems, gullible Bayes, K-Nearest neighbor and diverse others. exploitation these ways numerous varieties of data will be found utilizing affiliation principles, grouping and bunching. By exploitation this we have a tendency to extract data that describes candidate's performance and everyone their details. Even with large measures of data, the principal endeavor is to manage them; bunch examination is to characterize the crude data in an exceedingly smart means. The alleged grouping is a gathering of physical or unique articles, as indicated by the level of closeness between them, separated into a few gatherings, and makes comparative data questions inside a gatherings of high likeness and various gatherings of information objects which are not comparable. Underneath I have plot a portion of the numerous reasons selection representatives are actualizing enlisting methodologies through internet based life.

Diversity - All of these channels offer differing results that enrollment specialists can channel by a wide scope of socioeconomics and profession level to coordinate their needs.

Referral Improvement - Often, the intensity of a worker referral empowers the employing administrator to get to extra assets before expediting the alluded competitor. By applying this strategy to online networking devices, web based life selecting can help enlisting supervisors can audit a competitor's resume, collaborator suggestion, survey abilities, past occupation profiles and expert references for believability to guarantee that the contract is a decent move.

Expands Visibility - Recruiting through web based life empowers organizations and employment searchers to construct their very own locale or discussion with vocation openings and filter through numerous channels for significant outcomes. Most social stages offer alternatives to channel, match openings for work contingent upon area, aptitude level, and titles, long stretches of understanding and compensation go.

Huge Reach - Social media apparatuses assist scouts with drawing in with huge crowds to get a thorough feeling of attributes, culture, understanding and character. As relational association areas move from the PC to the PDA, mastermind information and correspondence can be composed into open space.

Online networking instruments and informal communication locales have reformed specialized strategies, both secretly and progressively, at work. Correspondence through web based life incorporates the usage of an online stage or webpage (a long range casual correspondence site) that enables people to pass on, generally speaking for a social explanation, through an arrangement of organizations, a huge segment of which are on the 
web and offer open entryways for people to interface over the web, for instance, for example by means of email and 'texting' (a type of ongoing, direct content based correspondence between at least two individuals utilizing PCs or different gadgets).

Enlistment through long range casual correspondence goals unequivocally, individual to individual correspondence areas (SNSs) empower customers to cause online profiles where individuals to can interface, using electronic life instruments. While at first intended for associating with loved ones, these systems have come to be viewed as a significant expert instrument, especially in the field of enrollment.

\subsection{Objectives}

- The aim of this project is to develop an SMAC Complaint Solution Suite for the recruitment industry In order to keep the enterprises updated with current technology and drive revenue through the digital eco-space.

- Review appropriate research by choosing qualitative or quantitative research methodologies in the field of Research.

- Examine, Expand, Re-evaluate the conceptual framework (or testing a theoretical model).

\section{EXISTING SYSTEM}

\subsection{Benefits of the Proposed Idea}

Recruiters have an instant advantage with LinkedIn simply because they're already used to searching for talent. Many have LinkedIn's premium version and are familiar with its HR platform and search functions. Since LinkedIn is a platform with thousands of professionals, recruiters are better at most at finding and sorting them, but from what I've seen that's about where their advantage ends.

\subsection{Opportunities}

Recruiters - here's the biggest opportunity I see for you... Being to use LinkedIn as a platform to rank your firm and your associates as experts with an in-depth wisdom. Giving valuable insights about salaries, career trends and job opportunities. Sure, you may be saying to yourself "that's what I do... That's who I am." Here's the problem - you're not successfully communicating this through a properly branded LinkedIn profile and focused activity which positions you as the Go-To person. Having gold LinkedIn Premium badge DOES'T build your brand, adding value for those you want to help and do business with DOES.

Ok, here's how I'm going to do this. First, LinkedIn is NOT a threat. Why? Because most companies that hire recruiters don't have all the time, resources and experience necessary to do it themselves. Most HR directors will quickly realize that staring at LinkedIn profiles on a computer screen all day isn't going to be the most productive use of their time, that's why they need you and your services. If you really think a social media platform itself can replace your entire industry... Then what does that say about the value you've built over all these years? Then again, if ALL you do is find anyone off the streets and put them in any job to get a commission check.... then maybe LinkedIn could replace you. Second, your biggest threat is YOU. Failure to really learn LinkedIn to this point means it is currently nothing more than a glorified search tool to find people with key words that pop up in your searches. I'm seeing even conservative industries like banking and financial services finally come around and invest in LinkedIn training. Maybe it's time for some recruiting companies to be honest with themselves.... And admit that successfully using LinkedIn is as challenging for you as it is for anyone else who is in sales. 


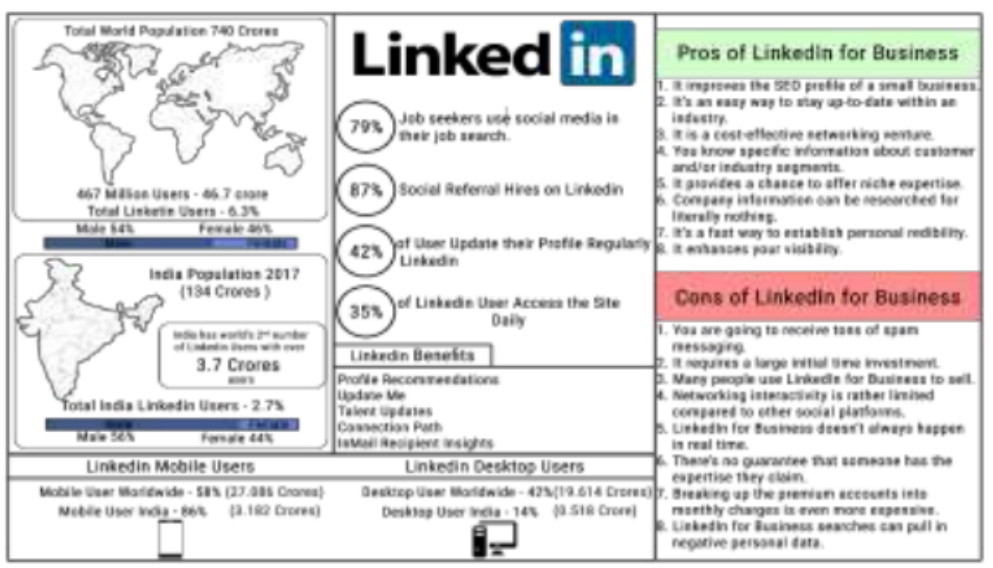

Figure 1 Linked In based social hiring statistics

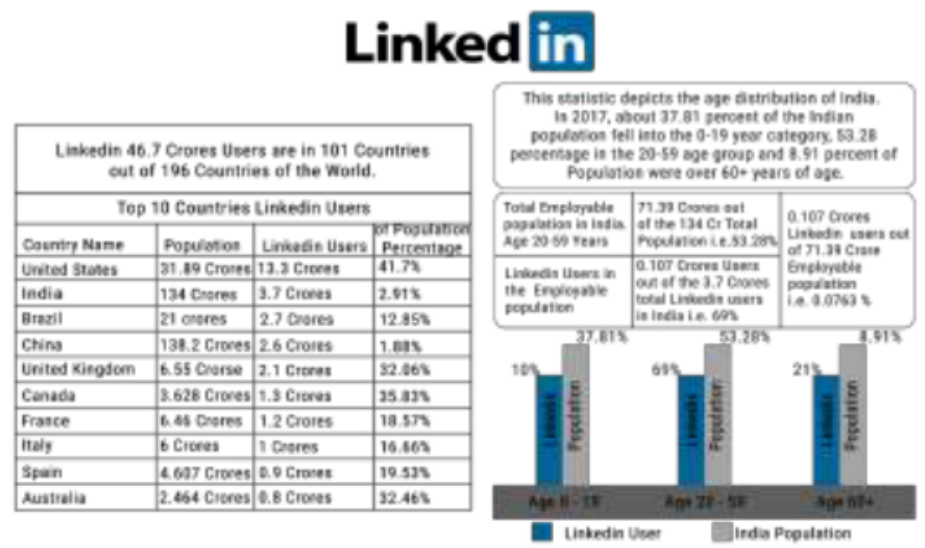

Figure 2 Linked population and other statistics

\section{SOLUTION ARCHITECTURE}

How does LinkedIn's search algorithm work?

There are four principle parts of the calculation. Calculations are acquainted with internet based life destinations as a method for lessening spam on the systems and making the substance important for the shoppers.

\subsection{Stage 1 - Initial Filter}

LinkedIn's automation system will arrange every single picture, content, long structure post and moreover video as one of three other options.

a) Spam

b) Low-Quality

c) Clear

I was not able source any substance online to discover how they choose what classification your post will be portioned into. I expect it is to do with the language that you are utilizing in the substance. For example, if you are using words, for instance, "offer", 
"headway", "markdown, etc. at that point it will be scored under the "spam" classification. I don't know of the definitions are for "Low-quality" or "clear". (In the event that you know, or have a thought, at that point please remark underneath)

\subsection{Stage 2 - Test Audience Process}

At the point when your post has been requested by the robots, it is then sent to an assurance of your group to see how conspicuous the substance is. It isn't conveyed to everybody that you are associated as well, only few your companions.

\subsection{Stage 3 - Scoring System}

At the point when put before a decision of your allies, different exercises have different loads in the count. For example, a "like" may simply have one point, where as a comment requires more effort, thusly, it has two points. A "share" shows that the substance is well known so will be allowed three points. (This is my variant of the scoring to clarify how it functions). The score of the post will choose its future or its destiny. It will either:-

a) Be downgraded on the grounds that it's low-quality

b) Be appeared to more individuals since it's high-caliber

\subsection{Stage 4 - Real People Assessment}

If you start to get responsibility on your post, by then it is passed along to "certifiable people at LinkedIn" who by then read each and every post and choose a decision concerning whether your substance ought to be conveyed to more individuals, or whether this is the stopping point. In the event that the substance is working truly well, at that point the Editors will likewise send the substance to individuals outside of your network. It will start to appear in "floating substance" on the application, or topical substance in the newsfeed, etc.

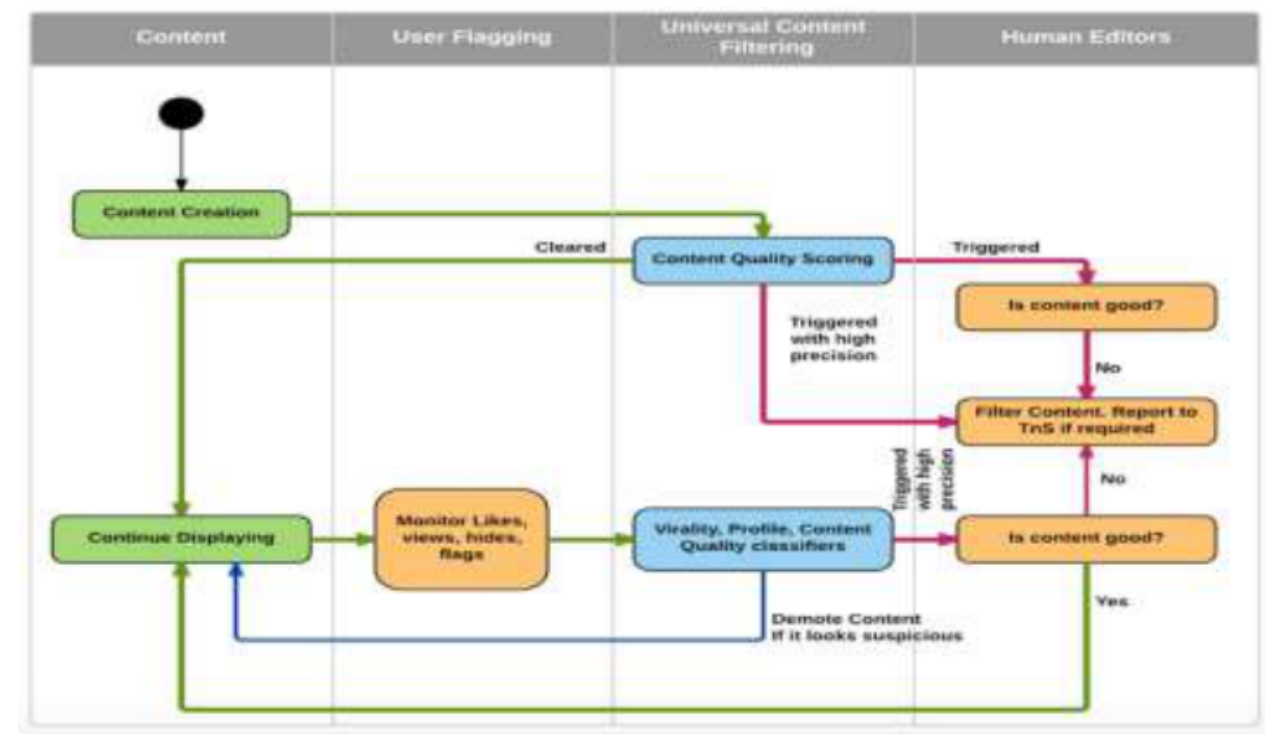

Figure 3 


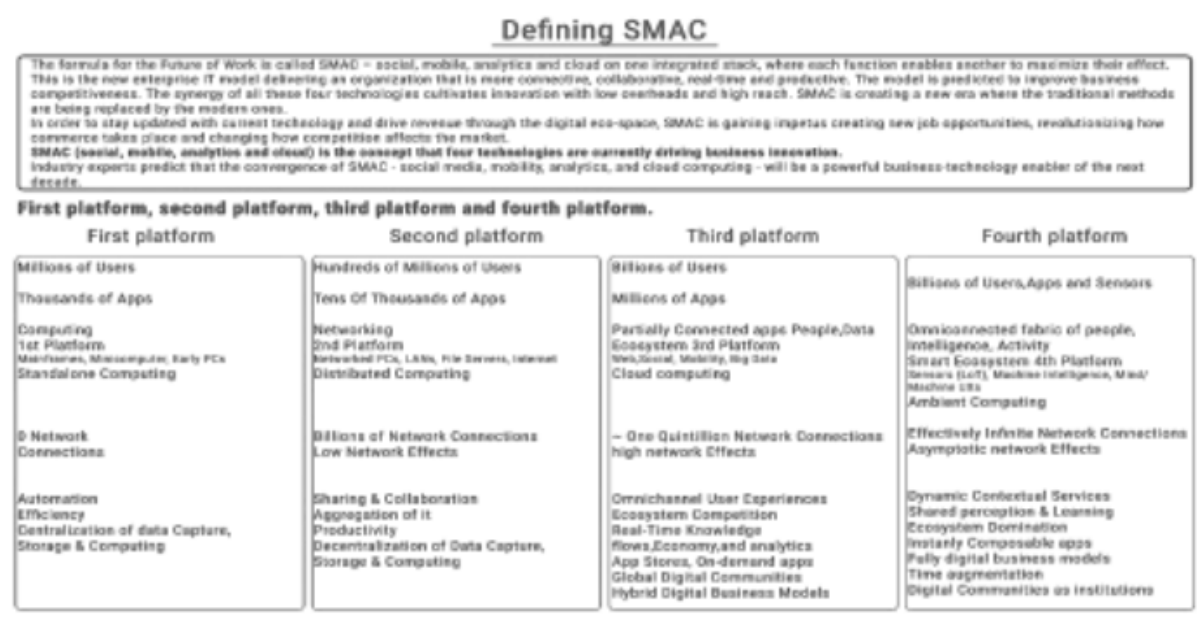

Figure 4 SMAC

The technologies under the SMAC umbrella are as follows:

Social: Web based life stages, for example, Twitter, Facebook, Instagram and Snap chat have given organizations better approaches to reach, collaborate with, target and get clients. It has offered ascend to new position titles, for example, web-based social networking influencer or advanced influencer, new promoting strategies, for example, viral showcasing efforts, and new information sources, for example, likes, reposts, hash tags and arrange associations.

Mobile: Versatile progressions and stages, for instance, the iPhone and the iPad, have changed the way wherein people pass on, shop and work. The introduction of related contraptions and wearable devices, the two of which rely upon unassuming sensors to make and transmit data, are the explanation behind new strategies and new organizations offered to customers.

Analytics: Information investigation enables organizations to see how, when and where individuals expend certain products and ventures. It is additionally utilized as a prescient marker for future client conduct just as when physical resources, for example, portions of a fly motors, will encounter debasement. As the expense for handling force and capacity diminished, examination turned into a top need for organizations. The open source venture Apache Hadoop introduced another period of examination called enormous information.

Cloud: Distributed computing gives another approach to get to innovation and the information a business needs to rapidly react to changing markets and take care of business issues. It introduced another approach to assemble foundation, stages and administrations. Amazon Web Services was one of the big disruptors in this space. 


\section{SMAC technologies}

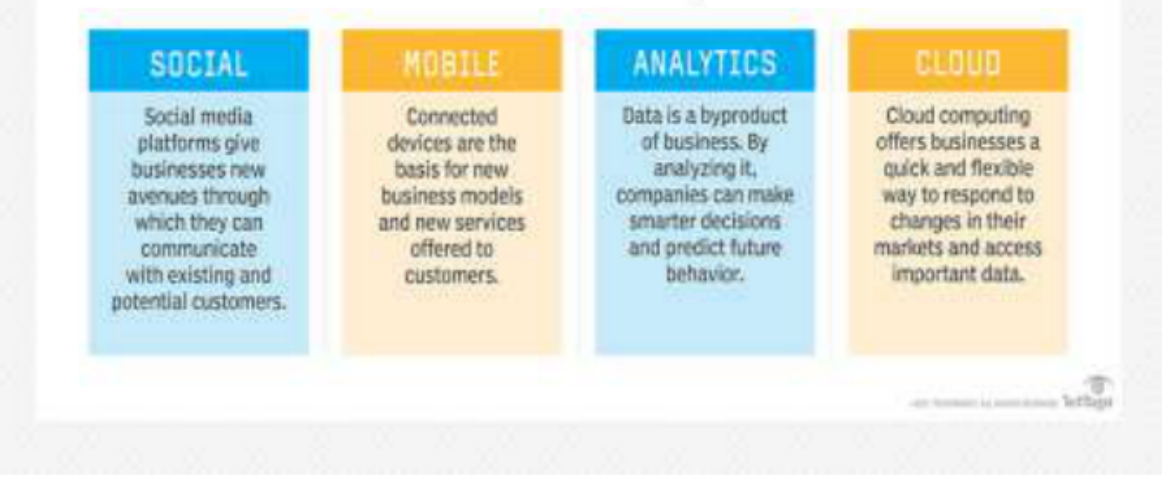

Figure 5 SMAC Technologies

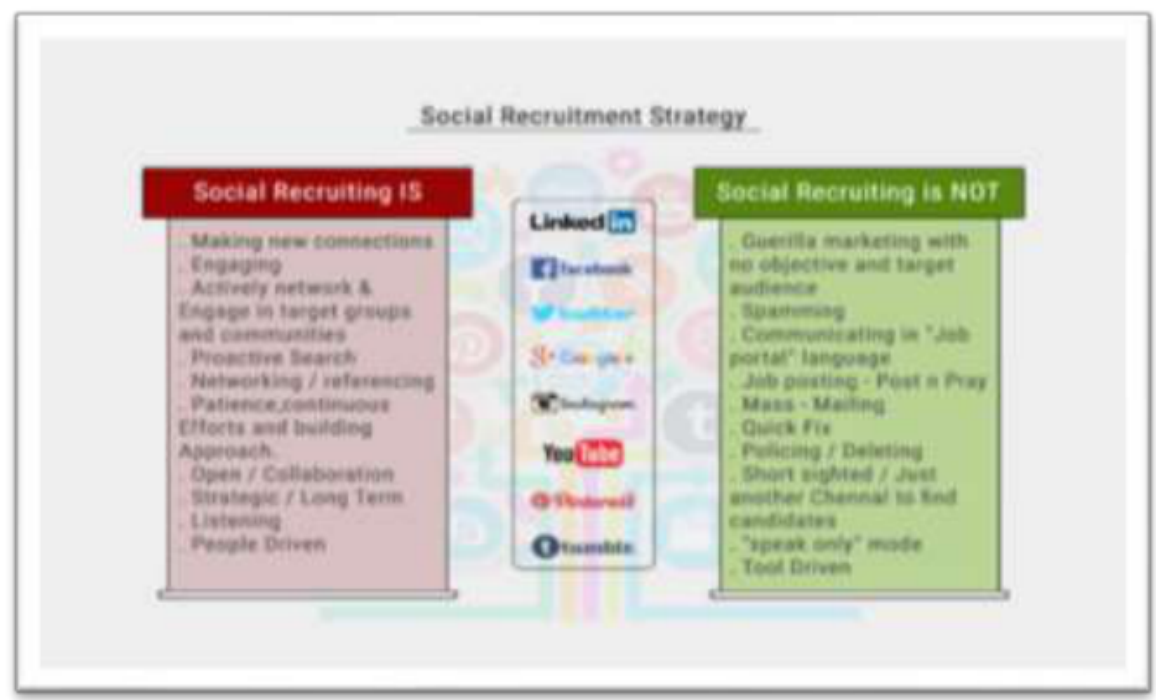

Figure 6 SRS

Discovering top ability in your field isn't constantly a simple assignment. Regularly, there's a huge incongruence between what organizations are searching for and what the work advertise brings to the table. Online networking can prove to be useful with regards to recognizing talented experts in the field and setting up an association.

Interface with the Right People

At one point, you'll need to take your web based life nearness to the following level. You'll need to begin interfacing with the correct individuals.

Keep your profiles industry-explicit and set up associations with industry pioneers, specialists in the field and individuals who have a specialty specialization. While doing as such, you'll need to individualize your methodology. You can't simply send one and a similar nonexclusive message to everybody, hoping to build up a strong network.

Convey individuals in a sufficient, credible way. Bid to their inclinations, in the event that you need to get the correct sort of reaction. Advise potential associations what you bring to the table and for what reason you'd prefer to stay in contact. Genuine, personalization requires time and exertion. On the off chance that you depend on nonexclusive correspondence, in any case, odds are that you'll pass up on numerous chances to associate with and possibly utilize experienced experts. 


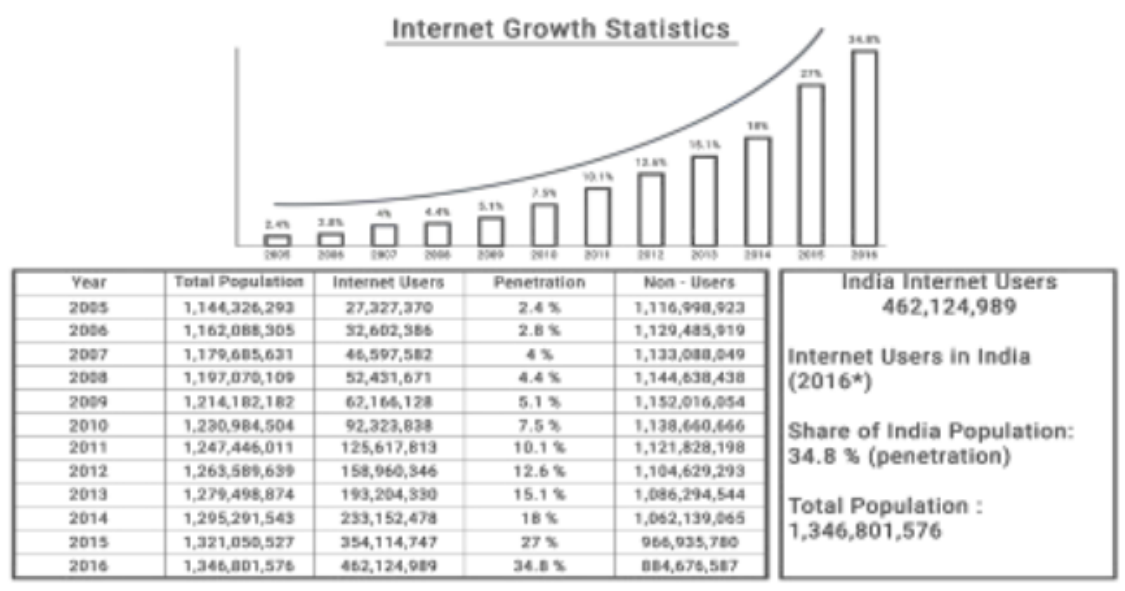

Figure 7 Internet Growth Statistics

With regards to contracting, it's only legitimate to take a shot at your LinkedIn profile. Measurements propose that 87 percent of spotters use LinkedIn, which makes it the top informal community of decision as far as finding talented competitors. Facebook is an inaccessible second with 55 percent and Twitter comes in third with 47 percent.

In spite of the fact that LinkedIn is a to some degree specific stage, its unmistakable quality in the realm of enrolling top ability will keep being high all through 2017.

One thing you can do to capitalize on your LinkedIn profile is update its initial 200 characters or thereabouts. Along these lines, individuals will realize that the organization is searching for new workers without navigating and arrive at the committed corporate page. Any individual who's keen on the message will along these lines get an opportunity to interface with the brand and investigate the work openings.

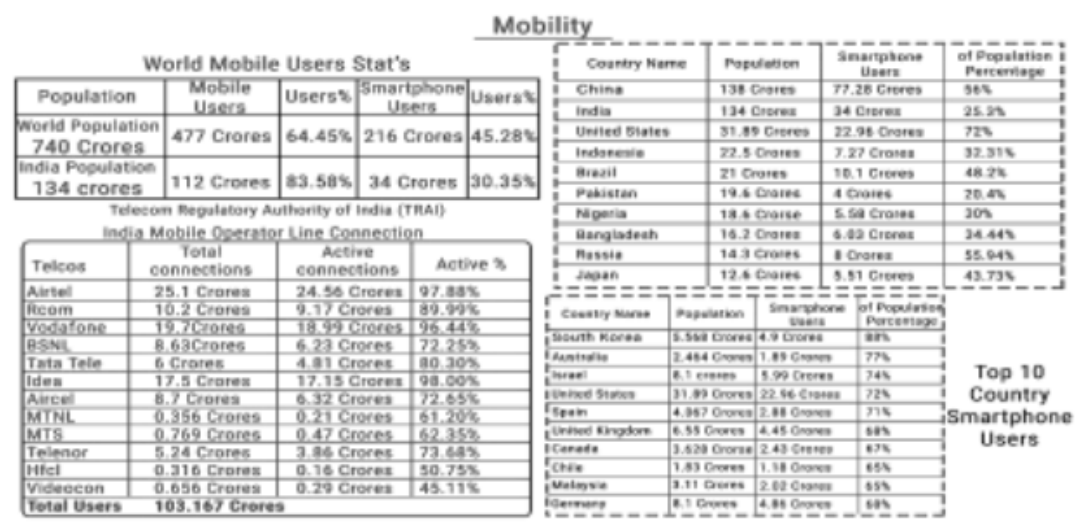

Figure 8

Cooperation in LinkedIn bunches is similarly significant. The informal organization has countless particular networks that pull in specialists in explicit fields. In case you're dynamic in pertinent gatherings, you'll see it a lot simpler to distinguish both top ability and influencers. Such data can be priceless with regards to drawing in the correct experts in an exceptionally aggressive specialty. 
The last choice to investigate is LinkedIn Pulse. Heartbeat offers you a chance to distribute an article on any point of intrigue. It very well may be profoundly particular, amusing or connected to your enlistment endeavors. Top notch Pulse distributions are distributed in the privilege LinkedIn gatherings, which expands their span.

\section{CONCLUSION \& FUTURE SCOPE}

In current modernized world, frameworks succeed out of the blue and their impact on different highlights on life is growing bit by bit. As the monetary log jam proceeds, a huge number of occupation searchers held by the activity butterflies are racing to join online interpersonal organizations in a scramble to manufacture their social capital. The examination led uncovers that greater part of respondents know about the long range informal communication destinations and there is low connection between's Age of business and the mindfulness about interpersonal interaction locales. One intriguing marker was that in current macroeconomic situation recurrence of changing employments has descended significantly. The examination likewise uncovers that the most well known systems administration site for looking for business is LinkedIn in light of its huge client database and one of a kind highlights like employment highlighter and specific email cautions. The utilization of Pulse can assist you with setting yourself as the authority inside the individual field. Building the correct notoriety is one of the keys to viable enlistment endeavors. Building up yourself as an impact in the separate specialty will augment acknowledgment and make it a lot simpler for the organization to interface with top ability.

\section{REFERENCES}

[1] Huang Tianchi, Li et al., "Design and Implementation of Living Streaming System Based on Multi-Service Nodes Collaboration", Advanced Information Technology Electronic and Automation Control Conference (IAEAC) 2017 IEEE.

[2] Daniel Yue Zhang, et al., "Towards Scalable and Dynamic Social Sensing Using A Distributed Computing Framework", 2017.

[3] Kusum Yadav and Kimaya Ambekar, SMAC Technologies Changing the Way we Travel, International Journal of Computer Engineering and Technology, 7(3), 2016, pp. 205-213

[4] Feng Xia, et al. "Socially Aware Conference Participant Recommendation with Personality Traits", 2017.

[5] Alexandru Topirceanu, et al., "UPT. Social: The Growth of a New Online Social Network", 2016.

[6] Ashish Jagtap, et al. "Homogenizing Social Networking with Smart Education by Means of Machine Learning and Hadoop: 2016.

[7] R. Vikram, G. Bhanu Prakash, G. Praneeth and Eshwar Reddy, A Frame Work to Improve Performance of Distributed Data Mining Using Ranking and Multi-Agent Systems. International Journal of Computer Engineering and Technology, 9(2), 2018, pp. 124-131

[8] Hong Wenxing, et al. A Mobile Reciprocal Job Recommender System", Computer Science \& Education (ICCSE) 2018.

[9] Yue, Yanling. "Analyzing Platform Evolution in the New Digital Era-Case Indoor Positioning in China." (2017).

[10] Özcan, Gözde,. "Applying Classifications Techniques in Job Recommendation System for Matching of Candidates and Advertisements." (2017).

[11] Ali Ikhwan, Milfa Yetri, Yohanni Syahra, Jufri Halim, Andysah Putera Utama Siahaan, Solly Aryza, Yasmin Mohd Yacob, A Novelty of Data Mining for Promoting Education 
Linkedin Talent Insights for Social Hiring in HR Recruitment using Data Mining Techniques using Social, Mobility, Analytics, Cloud (SMAC) Architecture

Based on FP-Growth Algorithm. International Journal of Civil Engineering and Technology, 9(7), 2018, pp.1660-1669

[12] Kasemsap, Kijpokin. "Multifaceted Applications of Data Mining, Business Intelligence, and Knowledge Management." 2018.

[13] Onik, et al.. "A recruitment and Human Resource Management Technique using Block chain Technology for Industry 2018.

[14] Masum, et al.. "Intelligent Human Resource Information System (i-HRIS): a Holistic Decision Support Framework for HR excellence (2018)

[15] Sawleshwarkar, et al.. "Simplified Recruitment Model Using Text-Mining on Psychometric and Aptitude Tests." IEEE, 2018.

[16] Şengül CAN and Mustafa GERŞIL, A Literature Review on the Use of Genetic Algorithms in Data Mining. International Journal of Computer Engineering \& Technology, 8(6), 2017, pp. 67-76

[17] Vetráková, Milota, et al. "Corporate Sustainability in the Process of Employee Recruitment through Social Networks in Conditions of Slovak Small and Medium Enterprises." 2018.

[18] Pandey, Jatin. "Managing Emotional Labor for Service Employees: An HRM-Based Approach." 2018

[19] Van Esch, et al. "Marketing Video-Enabled Social Media as Part of your E-Recruitment Strategy: Stop Trying to be Trendy." 2018. 